\title{
FLORA TIENDA DE FLORES: ENTREGAS DIFERENTES PARA CREAR UNA EXPERIENCIA DE SERVICIO INNOVADORA
}

\section{FLORA FLOWER STORE: DIFFERENT DELIVERIES TO CREATE AN INNOVATIVE SERVICE EXPERIENCE}

JEL Classification: M30, M31

Received: November 5, 2019 | Accepted: January 27, 2020 | Available Online: February 28, 2020

Cite this article as: Palacios-Chacón LA., Ortiz M., Giraldo M. (2019). Flora tienda de flores: entregas diferentes para crear una experiencia de servicio innovadora. Estudios de Administración, 26 (2), 38-51. https://doi.org/ 10.5354/0719-0816.2019.56950

\section{Lorena A. Palacios-Chacon \\ Instituto Tecnológico y de Estudios Superiores de Monterrey (ITESM), México \\ Universidad del Norte - Escuela de Negocios, Colombia \\ lorenapalacios@uninorte.edu.co - lapalaciosch@tec.mx \\ Mauricio Ortiz Velásquez \\ Universidad del Norte - Escuela de Negocios, Colombia mortiz@uninorte.edu.co \\ Mario Giraldo \\ Universidad del Norte - Escuela de Negocios, Colombia \\ mgiraldo@uninorte.edu.co}

\section{Resumen}

FLORA Tienda de Flores era, en el 2018, una floristería ubicada en la localidad ${ }^{1}$ de Riomar en Barranquilla, Colombia. Durante ese mismo año Andrea Cabarcas Castro, su fundadora, estaba preocupada por el crecimiento futuro de la empresa. Ella pensaba en estrategias para mejorar los puntos débiles que había detectado y tenía claro que debía mantener sus factores diferenciadores. La entrega de los arreglos al consumidor final representaba una oportunidad de mejora: este es el momento en el que se podía perder el control detallado de la experiencia de servicio FLORA por incumplimientos en los protocolos. Lo anterior podría afectar el posicionamiento positivo de la marca, podría llevar a una mala reacción de los consumidores en la experiencia post-compra, derivando así en un impacto negativo en los resultados financieros. Era octubre de 2018 y Andrea tenía hasta diciembre de ese mismo año para tomar una decisión. Su principal objetivo era mantener el crecimiento de la empresa, sin efectos negativos en la prestación del servicio.

1 En Colombia, las localidades son las divisiones administrativas con homogeneidad relativa desde el punto de vista geográfico, cultural, social y económico de los distritos especiales. Las localidades están establecidas por la ley 768 de 2002, la cual tiene por objeto "consagrar las normas que integran el Estatuto Político, Administrativo y Fiscal de los Distritos Especiales de Barranquilla, Cartagena de Indias y Santa Marta”. Barranquilla cuenta a 2019 con 5 localidades. 
Palabras claves: Servicios; Experiencia del cliente; Marketing; Colombia.

\section{Abstract}

FLORA Flower Shop was, in 2018, a flower shop located in the zone Riomar in Barranquilla, Colombia. During that same year Andrea Cabarcas Castro, its founder, was worried about the future growth of the company. She was thinking of strategies to improve the weaknesses she had detected and was clear that she should maintain FLORA's differentiating factors. The delivery of the floral arrangements to the final consumer represented an opportunity for improvement: This is the time when detailed control of the FLORA service experience could be lost due to protocol breaches. This could affect the positive positioning of the brand, lead to a bad reaction of consumers in the post-purchase experience, resulting in a negative impact on financial results. It was October 2018 and Andrea had until December of that same year to decide. Its main objective was to maintain the growth of the company, without negative effects on the delivery service.

Keywords: Services; Customer experience; Marketing; Colombia.

\section{Historia de la Empresa}

La Floristería FLORA Tienda de Flores, se fundó en octubre del año 2016 en la ciudad de Barranquilla, Colombia. FLORA, de acuerdo con sus clientes, logró convertirse en el año 2018 en una Floristería innovadora, posicionamiento que apenas con dos años de existencia en la ciudad había logrado gracias a sus diseños exclusivos (FLORA Box) con marca registrada y a través de nuevos diseños basados en la combinación de flores tipo exportación y bases de madera, así como de earthpack² (ver Imagen 1).

Imagen 1. FLORA Box

Fuente: FLORA Tienda de Flores

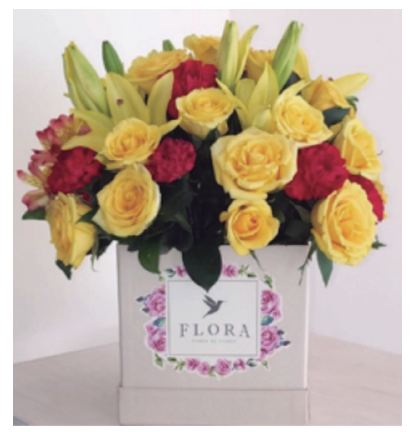

2 "Cartulina sin químicos blanqueadores, diseñada para empaques impresos que buscan comunicar un mensaje de compromiso con el medio ambiente, ideal para empresas con responsabilidad social cuyo fin es suministrar productos sostenibles de alto desempeño y óptimas condiciones de impresión”. http://www. propandina.com.ec/earthpact.html 
FLORA nació de la motivación de un emprendimiento liderado por Andrea Cabarcas Castro, quien después de haber ocupado puestos de dirección comercial y de marketing en empresas líderes de la región Caribe colombiana, decidió establecer su propia empresa en el sector de la comercialización de flores.

Gracias a la tradición familiar del consumo de flores de manera regular en la casa materna, la fundadora se dedicó a conocer más del sector de la comercialización de este producto. Ella estaba segura en el interés por emprender, pero además de la anterior idea, Andrea contaba con dos alternativas adicionales: producción y comercialización de snacks a base de frutos secos, y montaje de una delicatesen. Después de indagar en las tres opciones, Andrea se inclinó por la floristería al identificar que una de las principales falencias que se presentaba en el mercado de los estratos socioeconómicos 4, 5 y 6 de la ciudad de Barranquilla, era que las floristerías ofrecían arreglos homogéneos con diseños tradicionales en forma de triángulos como se observa en la Imagen 2.

Imagen 2. Arreglos Tradicionales en Triangulo

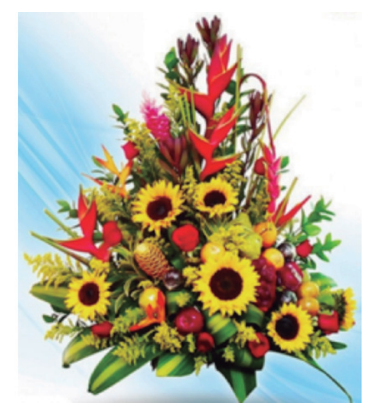

Fuente: Floristería Jardín Tricolor

https://jardintricolor.co/producto/xf-150/

FLORA inició sus labores con un equipo de cinco colaboradores que se desempeñaban (dos de ellos) en cargos administrativos y comerciales, así como en las áreas de armado y transporte. En un principio FLORA se dedicaba a vender flores al detal y arreglos para fechas especiales; en estos, además de ofrecer arreglos con diseños previamente establecidos por la floristería, también se le permitía al cliente participar en la creación de los mismos a través de la selección de la combinación de flores y complementos por parte de ellos. Con el correr del tiempo, monitoreando el mercado, la empresa fue ampliando su portafolio de productos y servicios, incursionando en la decoración institucional y de eventos especiales. La venta de flores al detal se daba en el punto de venta de la floristería en el que se tenían las flores y las bases para la elección de los clientes; los arreglos para fechas especiales se ofrecían a través de los medios digitales, como Instagram y Facebook; la decoración institucional estaba conformada principalmente por arreglos florales de oficinas, lobbies de edificios y reuniones empresariales; $y$, finalmente los eventos especiales que requieren diseños de espacios, así como de arreglos florales y montajes especiales.

En este sentido, gracias al incremento de las ventas de FLORA en el año 2018, el equipo de trabajo aumentó de 5 a 9 de la siguiente manera: dos administrativos, una persona adicional para armado y otra en apoyo a las entregas. Dada la oferta de valor en servicio que buscaba FLORA, las características fundamentales de los empleados incluían sensibilidad, empatía, excelentes relaciones con los clientes y con un alto 
sentido estético, elementos también inmersos en la misión, visión y slogan de la empresa.

\section{Misión, Visión y Slogan de la Compañía}

Como se mencionó con antelación, en el proceso de concepción de FLORA, como proyecto emprendedor, se identificó un conjunto de oportunidades que presentaba el mercado de Barranquilla. En este sentido los socios fundadores, en cabeza de Andrea Cabarcas, determinaron la realización de grupos focales de quienes se convertirían más adelante en el mercado objetivo de FLORA, estos grupos focales estaban conformados por hombres y mujeres entre 28 y 58 años que vivían en la Localidad Riomar (ver Figura 1).

Figura 1. Localidad de Riomar

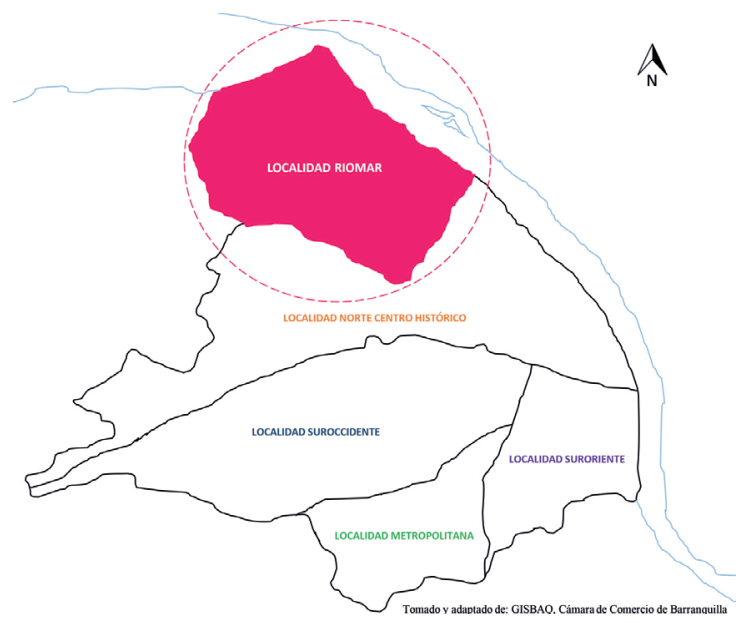

Fuente: Elaboración propia a partir de: GISBAQ, Cámara de Comercio de Barranquilla

Como resultado de esta labor se lograron identificar los elementos primordiales en los que se basaría FLORA para poder diferenciarse del mercado, a saber: 1 . Creación de una oferta de valor orientada al consumidor, 2. Orientación a la co-creación de servicio con el cliente, 3 . Diseño de la tienda con el fin de proveer experiencias memorables. Para el primero se pensó en ofrecer al mercado un alto nivel de compromiso en los tiempos de entrega y una eficiente atención a través de los medios digitales de comunicación; para el segundo, ofrecer la oportunidad de diseñar y crear con los clientes los arreglos florales de acuerdo con sus gustos, deseos y necesidades permitiéndoles hacer parte del armado de los mismos y facilitar la forma de pago al igual que coordinar cooperativamente las entregas de los arreglos; y, para el último elemento, el ambiente de la tienda (ej. aromas, colores, música, ambiente, decoración), la presentación de los empleados, y todos los elementos corporativos de la marca debían lograr, a través de los diseños y el servicio, inspirar en fechas importantes, encantar a personas especiales y/o enamorar a quienes se les desease manifestar sentimientos de amor.

De lo anterior se derivaron también la misión, la visión y el slogan de la empresa:

Misión: Inspirar, encantar y enamorar a través de una experiencia floral inolvidable. 
Visión: Ser reconocida en el 2021 como una las floristerías líderes en la ciudad de Barranquilla.

Slogan: Inspira, Encanta y Enamora.

Después de destacar la razón de ser y la perspectiva de FLORA, a continuación se exponen algunos elementos relevantes del mercado en el que se encuentra la compañía.

\section{Mercado de flores en Barranquilla}

Barranquilla es la cuarta ciudad más grande de Colombia, después de su capital Bogotá, Cali y Medellín. Como características sociales podemos encontrar una generalizada orientación al esnobismo y la ostentación en sus diferentes clases sociales, por lo cual el valor simbólico de la marca (ej. buen gusto, deseado) y el ser una marca reconocida favorablemente por los miembros de la sociedad es muy relevante para una empresa como FLORA.

Por otro lado, el mercado de las flores en Barranquilla, estaba constituido principalmente por tres tipos de consumidores, los primeros que regalan arreglos florales en fechas especiales; los segundos que compran flores para su consumo habitual (ej. Decoración en el hogar); y, los terceros que adquieren las flores para eventos. La forma de adquirir los productos y servicios por parte de los consumidores se da a partir de la compra de las flores al visitar las floristerías o al realizar los pedidos vía telefónica o por redes sociales, para que después sean entregados a domicilio. A su vez, las ventas dependían de las fechas especiales y ritos culturales en la región, a saber:

$\checkmark$ Fechas especiales: San Valentín (14 de febrero), día de la mujer (8 de marzo), día de la secretaria (26 de abril), día de la madre (segundo domingo de mayo), día del padre (segundo domingo de junio), amor y amistad (segundo fin de semana de septiembre) y navidad (24 de diciembre).

$\checkmark$ Ritos culturales (ocasiones especiales): cumpleaños, aniversarios, velorios, agradecimientos, reconciliación, matrimonios, baby showers, nacimientos y bienvenidas.

En este contexto y como será explicado en la sección posterior, varias floristerías tradicionales atendían el mercado de la ciudad vendiendo un producto y prestando un servicio estandarizado; es aquí donde FLORA logra destacarse por la diferenciación en sus arreglos incluyendo una extensa variedad de flores (tipos y colores principalmente pastel) e incluyendo modernas bases.

\section{Competencia}

La competencia directa de FLORA, aquella también enfocada en los estratos 4, 5 y 6 de la localidad de Riomar, estaba conformada por cinco floristerías a saber: Flores Express, Flores del Prado, Flor de Liz, Jardín de Alá y Mi Jardín de Flores (ver Figura 2). Por otro lado, la competencia indirecta estaba compuesta por 4 empresas más: Jardín Americano, Jardín Tricolor, Floristería Diana y Floristería D’Flores, las cuales si bien no se ubicaban en la localidad de Riomar eran reconocidas por su tradición en la ciudad (ver Figura 3). De las primeras cinco, dos de ellas ofrecían servicios similares a los de 
FLORA, principalmente la venta de flores al detal y arreglos para fechas especiales (Flores Express y Flores del Prado), las otras tres adicionalmente decoraban eventos especiales. Las cuatro indirectas se dedicaban a comercializar flores al mayoreo, hacer arreglos para fechas especiales y a realizar eventos.

Figura 2. Competencia Directa

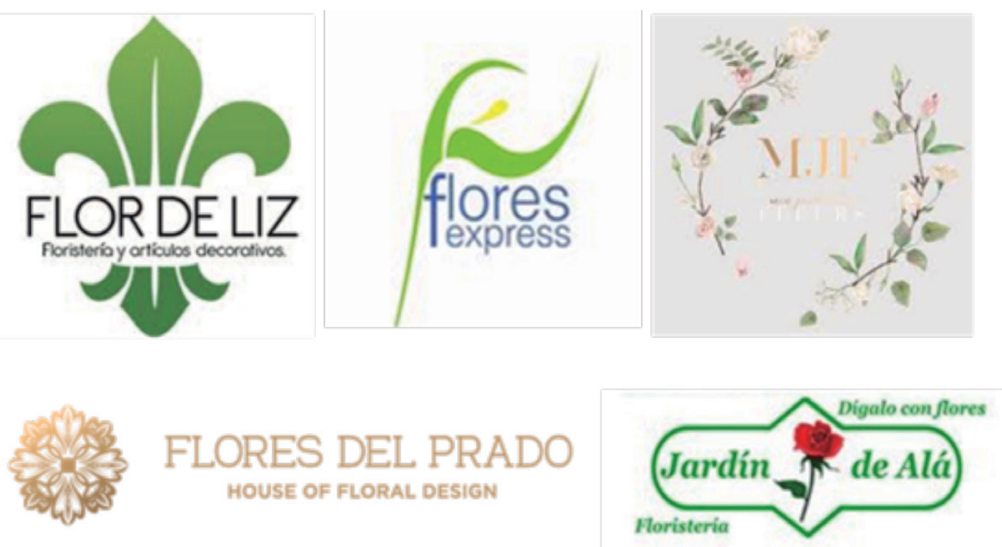

Fuente: http://www.flordeliz.com/; https://www.instagram.com/flores_express/?hl=es-la; https://www.facebook.com/mjardindeflores/?ref=py_c; http://www.floresdelprado.com/; http:// www.floristeriajardindeala.com/

Figura 3. Competencia Indirecta
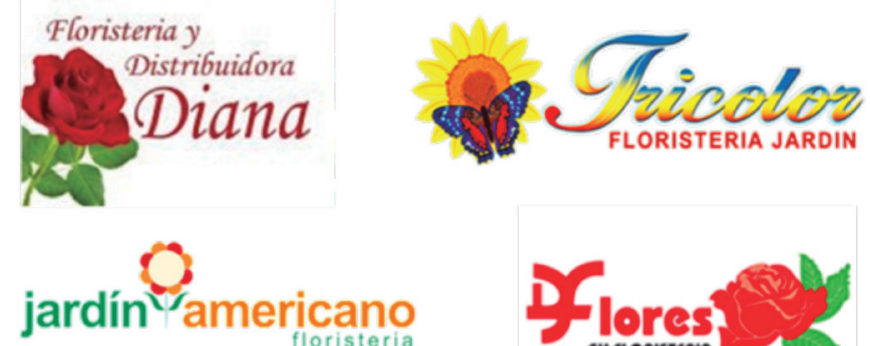

floristeria

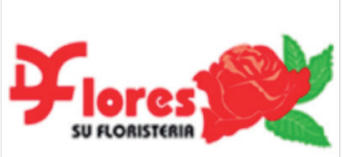

Fuente: http://floristeriadiana.com/es/?gclid=EAIaIQobChMIr7n-8KOE4QIVRR-

GCh0vxACpEAAYASAAEgKUnPD_BwE; https://es-la.facebook.com/floristeriajardintricolor/; http://tujardinamericano.com/; https://floristeriadflores.com/

Teniendo en cuenta las similitudes y diferencias con la competencia, en FLORA se destacan las características de co-creación de valor, innovación y servicio como sus 
ventajas competitivas; a continuación se resaltan los elementos más importantes a este respecto.

\section{Características de la experiencia de servicio Flora}

Las características del servicio en FLORA vienen dadas principalmente por la atención al cliente y el cumplimiento en las entregas, enmarcándose no solo en satisfacer las necesidades y deseos de éste, sino también en ofrecerle una experiencia diferente e innovadora a través de una atención personalizada y el respeto por el horario establecido en los domicilios (rangos u horas específicas), sorprendiendo al cliente y procurando un posicionamiento claro de la marca FLORA en el mercado, a través de la calidad de sus arreglos (durabilidad de las flores, exclusividad de la marca y diseño de los arreglos) así como en el cumplimiento en el servicio. El Cuadro 1 muestra los atributos más importantes para una compra en ocasiones especiales (en orden de importancia), de acuerdo con un análisis de los clientes de FLORA, hecho por Andrea.

Cuadro 1. Atributos más importantes para una compra en ocasiones especiales

\begin{tabular}{|l|c|}
\hline Atributos & Importancia (ranking) \\
\hline Belleza de los arreglos & 1 \\
\hline Diseño diferente con muy buen gusto & 2 \\
\hline $\begin{array}{l}\text { Innovador en la combinación de los tipos de flores y colores de } \\
\text { estas }\end{array}$ & 3 \\
\hline Cumplimiento en los tiempos y horas de entrega & 4 \\
\hline Excelente servicio en atención y asesoría & 5 \\
\hline Alta calidad de las flores & 6 \\
\hline
\end{tabular}

Fuente: elaboración propia con datos suministrados por la empresa

Cada uno de los clientes de FLORA tiene una forma particular de solicitar y adquirir las flores y/o los arreglos florales, ya sea visitando el punto de venta (en el que son atendidos por el equipo de administración y armado en cabeza de Andrea), realizando los pedidos vía telefónica o WhatsApp, así como a través de los diferentes canales digitales (Instagram y Facebook); ya se había diseñado una página web, pero aún no se había lanzado al mercado. Ahora bien, para la entrega de los arreglos florales la mayoría de los clientes, en un 90\%, prefieren el domicilio, que se llevan a cabo en la SUV (Small Urban Vehicle) de la fundadora (quien además hace entregas personalizadas) y en los motorizados subcontratados. El restante 10\% prefiere recogerlos en la floristería.

En cuanto al equipo colaborador de FLORA, este se caracterizaba por ser un equipo de trabajo cohesionado que se enfocaba en los objetivos de servicio y calidad de la floristería (alineado con la misión, la visión y el slogan), que se preocupa además por impregnar en cada arreglo el sentir de los clientes y así inspirar, encantar y enamorar a través de los diseños, colores, tipo de flores y arreglos (Ver Imagen 3). 
Imagen 3. Tipos de Arreglos Florales

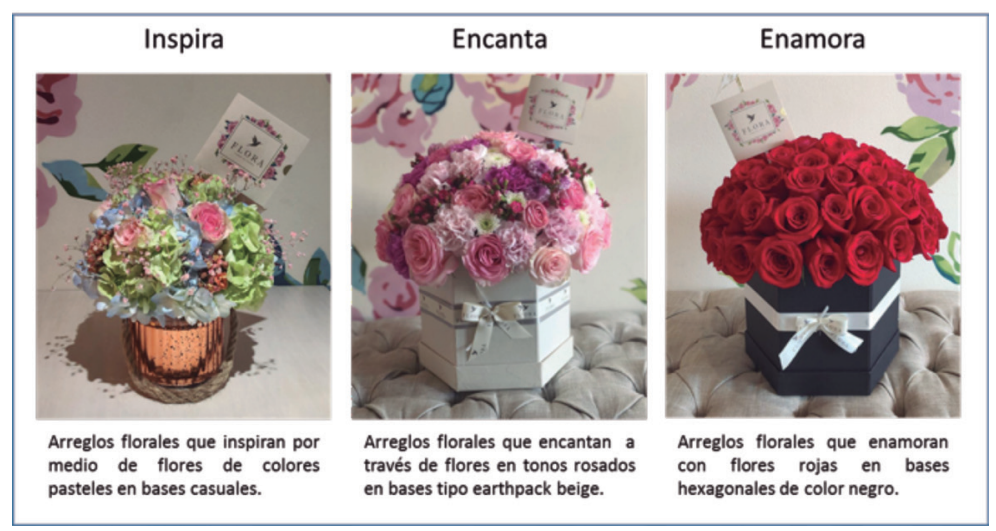

Fuente: FLORA Tienda de Flores

El tipo de liderazgo en FLORA era de carácter participativo, el cual lograba vincular a todos y cada uno de los colaboradores implicados en el proceso desde la toma de pedido, pasando por el armado, llegando hasta el momento de la entrega. Por lo tanto, se les permitía y se les invitaba, desde la dirección de la fundadora, a que participaran en los procesos y lograran plasmar en los arreglos los deseos y necesidades de los clientes, así como en asimilar e interpretar las preferencias de los diseños antes mencionadas y sus requerimientos de entrega en cuanto a hora, lugar y fecha en particular.

El equipo de FLORA estaba conformado, desde el momento en el que se vinculaban a la floristería, por un grupo de seres humanos sensibles a los que se les remuneraba en un porcentaje superior al promedio de salarios de enganche del sector. De igual manera, se les capacitaba año a año, con expertos nacionales y regionales, en diferentes técnicas de arreglos y decoración. Finalmente, las directivas de la floristería propendían por el equilibrio en las horas de entrada, salida y turnos entre los colaboradores para que estos pudieran atender sus necesidades personales y ocupaciones familiares.

Desde la creación del concepto, basado en el estilo de decoración Shabby Chic Vintage para cumplir con el slogan o la promesa que FLORA ofrecía a sus clientes de Inspirar, Encantar y Enamorar, Andrea lograba presentar arreglos diferentes gracias a la combinación de los colores (pasteles principalmente), las formas de los arreglos (orgánicos, sembrados e irregulares), los tipos de flores (hortensias, garberas y rosas) que se conservaban en el cuarto frío de la floristería, así como las bases (madera y earthpack) en los que se hacían los arreglos. Este cuarto frío, diseñado y construido específicamente para FLORA, contaba con las especificidades adecuadas de humedad y temperatura para la conservación de las flores.

De igual manera Andrea establecía, de acuerdo con las necesidades y deseos de los clientes, los precios para cada uno de los productos y servicios de la floristería, así como la manera de cómo llegar y cómo comunicarse con sus clientes potenciales y reales; para esto Andrea encontró en el marketing digital la mejor opción. En cuanto a la recuperación del servicio, la emprendedora se encargaba directamente de las devoluciones, si alguien no estaba contento con alguno de los arreglos, podía pedir 
otro a cambio sin cuestionamientos, lo único importante era preguntar por las razones de la devolución como un elemento de aprendizaje para la empresa y se ofrecía algo adicional a ese cliente (ej. Bono de regalo, envío gratis en una próxima compra, un arreglo gratuito para una ocasión específica).

En cuanto al punto de venta, este era de fácil acceso, se ubicaba en una zona de tráfico fluido y contaba con parqueadero privado. FLORA es una Tienda de Flores (ver Imagen 4) no es un local que vende flores, no es una casa adaptada para vender flores, todo lo contrario, es un lugar diseñado y concebido de manera exclusiva para brindar una experiencia diferente, un espacio en el que se encuentren los clientes y las flores para crear arreglos únicos e innovadores. Cada uno de los elementos ornamentales y de diseño de la Floristería, así como los uniformes de cada uno de los colaboradores y los elementos comunicacionales (tarjetas de presentación, tarjetas de mensajes, logos en las bases y la cuenta de Instagram) de la floristería responden al Manual de Imagen Corporativa de la misma (ver Imágenes 5, 6, 7 y 8).

Imagen 4. Interior de FLORA Tienda de Flores

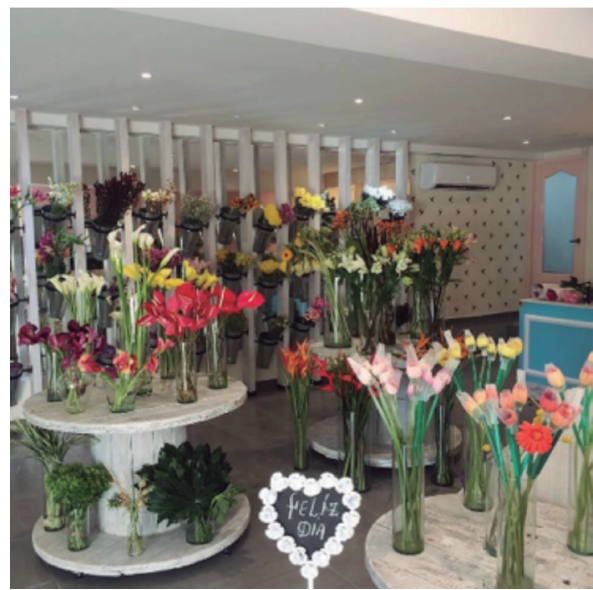

Fuente: FLORA Tienda de Flores

Imagen 5. Logo de FLORA

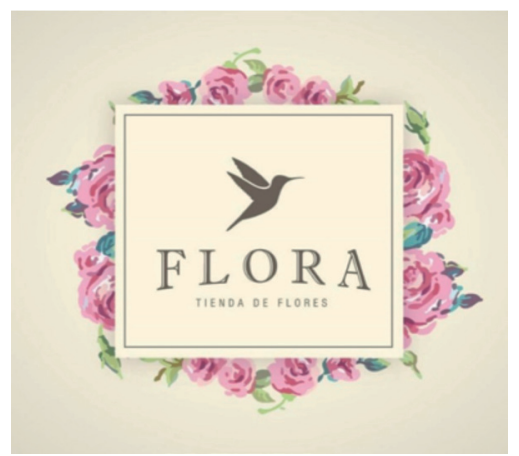

Fuente: FLORA Tienda de Flores 
Imagen 6. Uniforme de FLORA

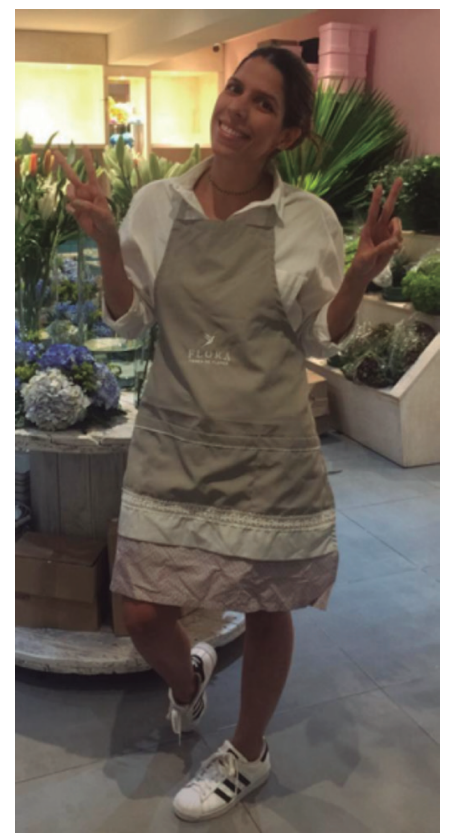

Fuente: FLORA Tienda de Flores

Imagen 7. Tarjeta de presentación de FLORA

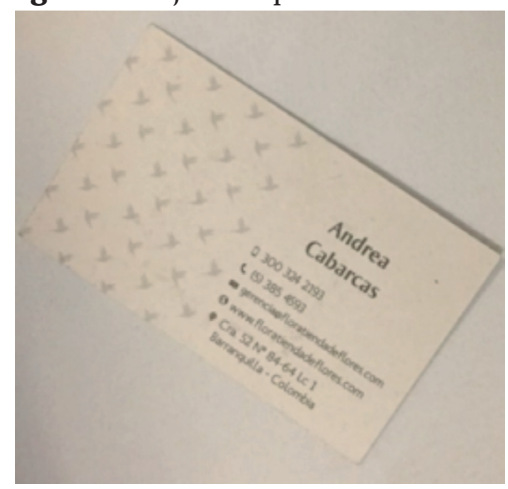

Fuente: FLORA Tienda de Flores 
Imagen 8. Tarjeta de mensaje de FLORA

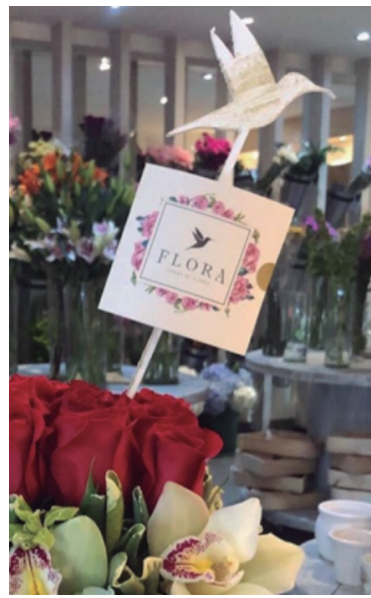

Fuente: FLORA Tienda de Flores

A manera de resumen, la experiencia de servicio de FLORA se refleja en las siguientes etapas:

\section{Pre-experiencia:}

Esta etapa depende de las fantasías de los clientes que se combinan con las expectativas de quien recibe y las razones por las cuales se otorga el regalo. Desde el punto de vista del servicio de FLORA, estos tres elementos se armonizan a través de las sugerencias de los empleados y de la empatía de los mismos. Lo anterior reforzado con la co-creación del arreglo y el esmero en la entrega para mantener la calidad.

\section{Proceso de compra:}

Vale la pena resalar que las diferentes etapas de experiencia de consumo de FLORA inician desde el momento en que los clientes que van a adquirir los arreglos florales entran en contacto a través de los diferentes medios digitales con los que cuenta flora, a saber: Instagram, Facebook y WhatsApp.

Luego, empieza el proceso solicitando arreglos específicos que buscan los clientes ya sean preguntando por los que ellos tienen en su mente o por lo que aparecen en redes sociales. Una vez en FLORA se recibe esa solicitud, la persona encargada responde de tal manera que trata de satisfacer los deseos y/o las necesidades del cliente y proponiéndole algunos ajustes que se le puedan hacer el arreglo dependiendo de la ocasión y del objetivo que el cliente tenga en su cabeza. Luego la persona encargada pasa la orden de pedido a la zona de armado donde se le otorga un turno con un número específico y se le ratifica de inmediato al cliente la hora que va a ser entregado el arreglo, se le solicita el pago para así pasar al proceso de armado. En esta etapa existe un catálogo donde están los diseños propuestos por FLORA y que además atienden las solicitudes adicionales del cliente.

Cuando el arreglo está listo se pasa a la zona de despacho para que la persona encargada de éste los organice de tal manera que se cumpla con los horarios de entrega en las condiciones adecuadas, ya sea que se vaya a entregar en una moto, en un servicio especializado o lo entregue la misma fundadora de la empresa. Estos 
arreglos se entregan, en el mejor de los casos a la persona que se les envía, en su defecto al familiar o empleado que lo reciba.

\section{Proceso de uso:}

El diseño y colores de las flores, así como la funcionalidad de las bases en las que se entregan, ayudan a resaltar los espacios de las casas, oficinas y eventos. Así, además de ser un buen detalle, pueden tener usos complementarios que complementan la experiencia.

\section{Reminiscencia:}

Esta etapa está relacionada con los recuerdos del cliente respecto a la experiencia FLORA. La Imagen 9 muestra como ejemplo algunos comentarios en Instagram, que es uno de los medios más usados para pedidos y promoción.

Imagen 9. Comentarios en redes sociales

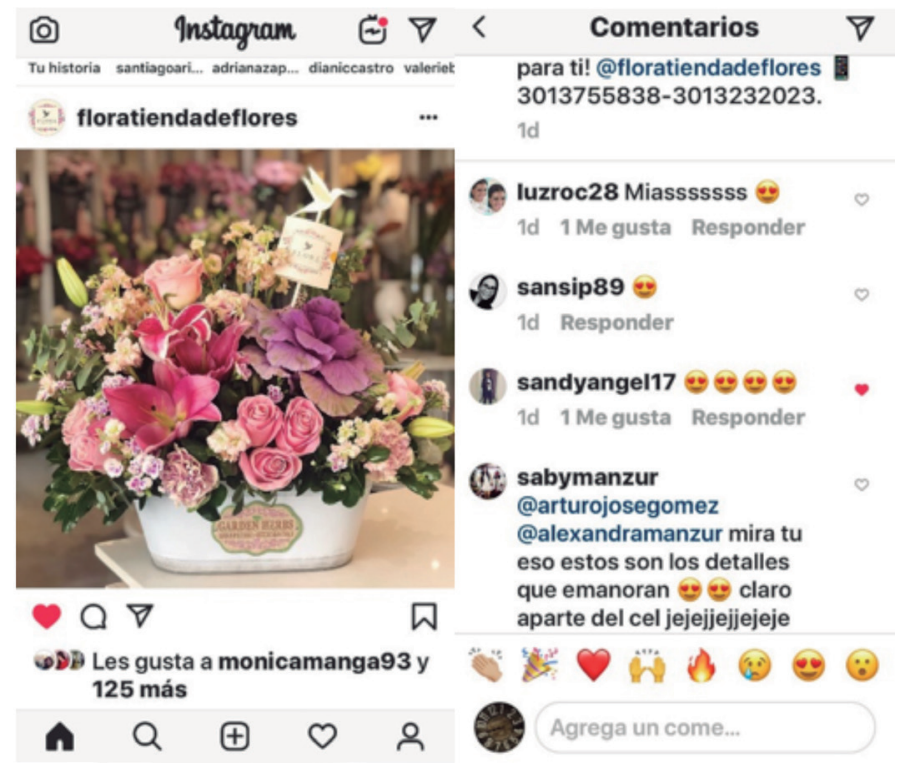

Fuente: datos suministrados por la empresa

\section{Cierre etapa proceso de compra y decisión a tomar}

Como se detalló en la sección anterior, FLORA Tienda de Flores llegaba hasta el 2018 de manera directa a un 50\% de su mercado a través de su propia red de distribución y logística que estaba conformada por una SUV de propiedad de la fundadora para hacer las entregas especiales que consisten en arreglos de gran formato. Además, contaba con un servicio subcontratado motorizado que transportaba los arreglos pequeños (25\% de las ventas); así como por un transporte especializado, también tercerizado, para hacer el $25 \%$ de las entregas restantes. Tanto las entregas realizadas por la SUV, el motorizado y el transporte especializado tomaban en promedio cada una de ellas entre 45 y 60 minutos para completar el servicio (ver Anexo 1). Ahora bien, es importante tener en cuenta que el aprovisionamiento de las flores, desde 
los diferentes puntos de acopio de la ciudad de Barranquilla (Aeropuerto y Terminal de Transporte) provenientes vía aérea y terrestre de ciudades de Colombia tales como Bogotá y Medellín, también se hacía en los vehículos citados anteriormente, a excepción del servicio motorizado.

Las entregas hechas por la fundadora permitían un contacto directo con quien recibía y un cierre adecuado del servicio. Cuando se optaba por un medio distinto, tal como moto o entrega tercerizada, algunas veces no se cumplía a cabalidad con el tiempo que se prometía, ni con el protocolo de entrega. Adicionalmente, en algunos casos los arreglos presentaban algún tipo de defecto porque se movían en el camino y antes de entregarlos no se ponía atención a los detalles. Finalmente, en ninguno de los dos casos mencionados se transmitía la cultura organizacional de FLORA, ni existía el esmero que ponía Andrea en hacer cada entrega diferente y especial.

Por el crecimiento de FLORA (ver Gráfico 1), por su proyección en el mercado y porque Andrea había detectado las fallas mencionadas en las entregas de los arreglos, debía tomar entonces una decisión que le permitiera: a. Mejorar su servicio de entrega (tanto en tiempo como experiencia); b. aumentar en un 10\% el número de arreglos entregados mensualmente; c. cubrir una mayor extensión geográfica en la ciudad de Barranquilla; y, d. disminuir costos de transporte en un 30\%. Era octubre de 2018 y teniendo en cuenta que para el fin de año Andrea se debía plantear la estrategia financiera y de servicio para el 2019, se encontraba en la disyuntiva de cuál sería la mejor opción para llevar la experiencia FLORA al nivel óptimo de servicio.

Gráfico 1. Crecimiento Número de Arreglos FLORA

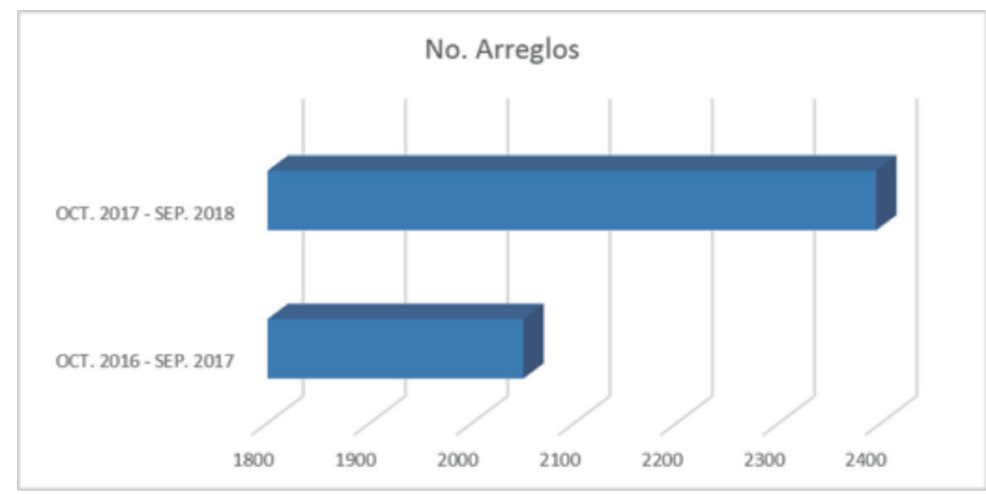

Fuente: FLORA Tienda de Flores

\section{Declaración de conflicto de interés}

Los autores del presente manuscrito manifiestan que no existen conflictos de interés con ninguna entidad o institución, ni de carácter personal en esta publicación. 


\section{Anexos}

Anexo 1. Tabla Comparativa Costos Transporte FLORA

\begin{tabular}{|c|c|c|c|}
\hline \multicolumn{4}{|c|}{ FLORA Tienda de Flores - Caso } \\
\hline \multicolumn{4}{|l|}{ Operación de las Entregas Actuales - 2018} \\
\hline Número de Entregas al mes: 300 & Tiempo de Entrega & Área Geográfica & Costos Entrega \\
\hline Costo de Entregas al mes & & & $\$ 3.225 .000,00$ \\
\hline SUV (150) & 45 Minutos & Barranquilla & $\$ 2.100 .000,00$ \\
\hline Moto (75) & 45 Minutos & Barranquilla & $\$ 525.000,00$ \\
\hline Especializado (75) & 60 Minutos & Barranquilla & $\$ 600.000,00$ \\
\hline \multicolumn{4}{|l|}{ Operación de las Entregas VAN Brandeada - 2019} \\
\hline Número de Entregas al mes: 300 & Tiempo de Entrega & Área Geográfica & \\
\hline Costo de Entregas al mes & & & $\$ 2.220 .000,00$ \\
\hline Financiación VAN - Brandeada & & & $\$ 600.000,00$ \\
\hline Conductor (1) & 30 Minutos & Barranquilla & $\$ 1.200 .000,00$ \\
\hline Mantenimiento VAN - Brandeada & & & $\$ 420.000,00$ \\
\hline \multicolumn{4}{|l|}{ Operación de las Entregas Motos- 2019} \\
\hline Número de Entregas al mes: 300 & Tiempo de Entrega & Área Geográfica & \\
\hline Costo de Entregas al mes & & & $\$ 4.100 .000,00$ \\
\hline Financiación de 3 Motos Brandeadas & & & $\$ 300.000,00$ \\
\hline Conductores (3) & 20 Minutos & Barranquilla & $\$ 3.600 .000,00$ \\
\hline Mantenimiento de 3 Motos Brandeadas & & & $\$ 200.000,00$ \\
\hline \multicolumn{4}{|l|}{ Operación de las Entregas Tercerizado - 2019} \\
\hline Número de Entregas al mes: 300 & Tiempo de Entrega & Área Geográfica & \\
\hline Costo de Entregas al mes & & & $\$ 2.100 .000,00$ \\
\hline Entregas Tercerizadas & 30 Minutos & Barranquilla & $\$ 2.100 .000,00$ \\
\hline $\begin{array}{l}\text { *Con la operación de la VAN se aprovecha la ir } \\
\text { brandeado, que si se contratara una empresa que } \\
\text { de } \$ 1.200 .000,00 . * * \text { Las Entregas y el tiempo, así } \\
\text { análisis del Caso. }\end{array}$ & $\begin{array}{l}\text { plemenatción de u } \\
\text { preste ese servicio } \\
\text { omo los costos aqu }\end{array}$ & $\begin{array}{l}\text { ategia de comur } \\
\text { slicidad móvil, te } \\
\text { sados, son valore }\end{array}$ & $\begin{array}{l}\text { n por su color y } \\
\text { in costo mensual } \\
\text { eferencia para el }\end{array}$ \\
\hline
\end{tabular}

Fuente: FLORA Tienda de Flores 